\title{
DIALYSIS
}

\section{Biocompatible versus standard PD solutions-more balANZ data}

A further analysis of balANZ study data has expanded on the previously observed association between use of biocompatible peritoneal dialysis (PD) fluid and a reduced risk of peritonitis, to demonstrate improvements in some peritonitis-related outcomes.

The balANZ study was an openlabel, randomized controlled trial in which 185 adults on PD were randomly assigned to use either a biocompatible PD solution (which has a neutral $\mathrm{pH}$ and is low in potentially nephrotoxic glucose degradation products) or a conventional PD solution for 2 years. The primary outcome results published earlier this year showed that use of biocompatible PD fluid did not slow the rate of renal function decline over conventional solution, although it did delay the time to anuria. In addition, biocompatible fluid did seem to reduce the incidence of peritonitis. The most recent publication aimed to determine whether the beneficial effects of the biocompatible PD fluid on peritonitis were specific for particular micro-organisms and whether biocompatible fluid was associated with improved peritonitis outcomes (such as hospitalization duration, severity, peritonitis-related technique failure, and peritonitis-related death).

The researchers found that rates of culture-negative, Gram-positive, Gramnegative, and polymicrobial peritonitis episodes were similar in the two groups, but that the rate of nonpseudomonal Gram-negative peritonitis was significantly lower in the biocompatible fluid group. Use of biocompatible fluids

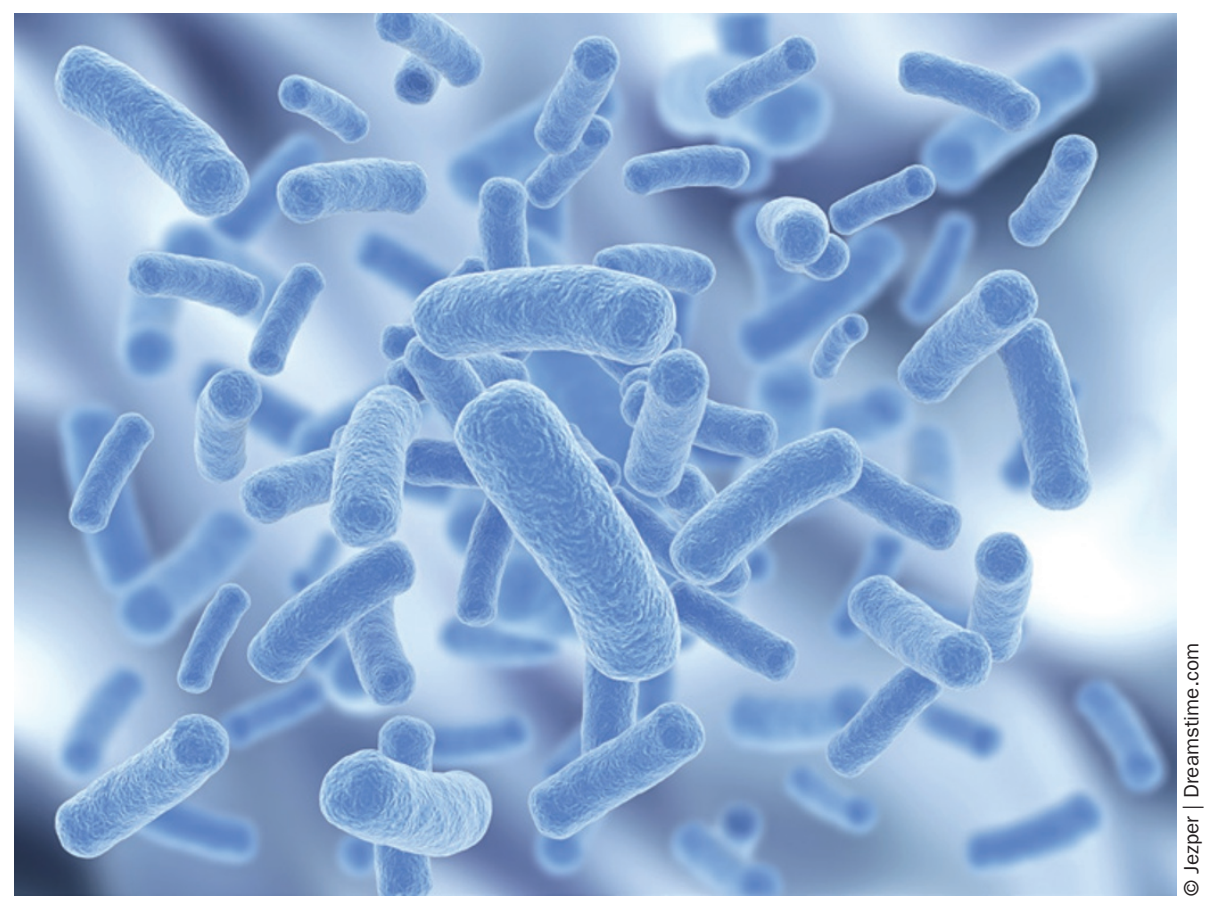

was not associated with a reduction in the risk of peritonitis-related hospitalization, but was associated with a shorter median hospitalization duration, and with peritonitis that was more likely to be rated mild. Rates of peritonitisrelated death and peritonitis-related technique failure did not differ between the groups.

"This is the largest randomized controlled trial comparing the effects of biocompatible PD fluids with conventional PD fluids on PD outcomes, and it demonstrated a reduced incidence of PD peritonitis and reduced severity of peritonitis when it did occur," says Fiona Brown, an author on the paper. "This is a very significant result as peritonitis is one of the most serious complications of PD. It is, however, important to note that this was not the primary end point of this study, and that conflicting results from other studies indicate that further clinical research in this area is vital to determine the exact benefits of biocompatible PD fluids."

Rebecca Kelsey

Original article Johnson, D. W. et al. The effects of biocompatible compared with standard peritoneal dialysis solutions on peritonitis microbiology, treatment and outcomes: the balANZ trial. Perit. Dial. Int. 32, 497-506 (2012) 